OPEN ACCESS

Edited by: Yun-Qing Li,

Fourth Military Medical University,

China

Reviewed by:

Adhil Bhagwandin,

University of Cape Town, South Africa

Dengshun Miao,

Nanjing Medical University, China

*Correspondence:

Li-Cai Zhang

licaizhang001@163.com

Received: 22 April 2020

Accepted: 22 July 2020

Published: 12 August 2020

Citation:

Song S-Y, Zhai X-M, Shan C-J,

Lu L-L, Hong J, Cao J-L and Zhang L-C (2020) A Special Cranial Nucleus (CSF-Contacting Nucleus) in Primates. Front. Neuroanat. 14:53.

doi: 10.3389/fnana.2020.00053

\section{A Special Cranial Nucleus (CSF-Contacting Nucleus) in Primates}

\author{
Si-Yuan Song, Xiao-Meng Zhai, Cheng-Jing Shan, Lei-Lei Lu, Jia Hong, Jun-Li Cao and \\ Li-Cai Zhang*
}

Jiangsu Province Key Laboratory of Anesthesiology, Xuzhou Medical University, Xuzhou, China

Background: There is a unique nucleus (CSF-contacting nucleus) in the brain of rat. It has been demonstrated in our previous research. The extraordinary feature of this nucleus is that it is not connected to any parenchymal organ but to the CSF. In primates, however, the presence or absence of this nucleus has not been proven. Confirmation of the presence of this nucleus in primates will provide the structural basis for brainCSF communication and help to understand the neurohumoral regulatory mechanisms in humans.

Methods: The tracer cholera toxin B subunit conjugated to horseradish peroxidase (CBHRP) was injected into the CSF in the lateral ventricle (LV) of primate rhesus monkeys. After $48 \mathrm{~h}$, the monkeys were perfused and the brain was dissected out, and sectioned for CB-HRP staining. The CB-HRP positive structures were observed under confocal and electron microscopy. The three-dimensional (3D) structure of the CB-HRP positive neurons cluster was reconstructed by computer software.

Results: (1) CB-HRP labeling is confined within the ventricle, but not leakage into the brain parenchyma. (2) From the midbrain inferior colliculus superior border plane ventral to the aqueduct to the upper part of the fourth ventricle (4V) floor, a large number of CB-HRP positive neurons are consistently located, form a cluster, and are symmetrically located on both sides of the midline. (3) 3D reconstruction shows that the CB-HRP positive neurons cluster in the monkey brain occupies certain space. The rostral part is large and caudal part is thin appearing a "rivet"-like shape. (4) Under electron microscopy, the CB-HRP positive neurons show different types of synaptic connections with the non-CSF-contacting structures in the brain. Some of the processes stretch directly into the ventricle cavity.

Conclusion: Same as we did in rats, the CSF-contacting nucleus is also existed in the primate brain parenchyma. We also recommend listing it as the XIII pair of cranial nucleus, which is specialized in the communications between the brain and the CSF. It is significant to the completing of innervation in the organism.

\footnotetext{
Keywords: CSF-contacting nucleus, primate, rhesus monkey, cerebrospinal fluid, innervation
} 


\section{INTRODUCTION}

The animal body consists not only of parenchymal organs, but also of fluids (such as brain interstitial fluid, plasma, and CSF). Physiology studies suggest that the levels and composition of the body fluids are also coordinated under the instructions from the brain (Agnati et al., 2017). The components of the plasma, CSF, and the brain extracellular fluid are different and these fluids are physically separated from each other (Rambeck et al., 2006). How does the central nervous system relay the information to these fluids and functionally modulate their levels and composition remains to be answered.

In the past 30 years, our studies revealed that after injecting peripheral nerve tracer CB-HRP into the rat ventricular system, the tracer is confined to the ventricular wall because of the existence of the brain barriers. The CB-HRP positive neuron cluster is always present at the ventral gray of the lower part of the aqueduct and the upper part of the $4 \mathrm{~V}$ floor (Zhang et al., 1994, 2003; Lu et al., 2008; Song et al., 2019). Only the neural processes that stretch into the CSF can be labeled. These neurons have a consistent location, form an independent cluster, occupy a certain space, and have a clear boundary with the nearby structures. The CSF-contacting neurons cluster is in accordance with the definition of the nucleus. Therefore, we name it as the "cerebrospinal fluid-contacting nucleus" or "CSF-contacting nucleus" (Song and Zhang, 2018; Song et al., 2019). Studies have confirmed that the CSF-contacting nucleus has broad synaptic and non-synaptic connections with the other neurons and the blood vessels, and has the morphology for sensing and modulating the body fluids (Zhang et al., 2003; Liang et al., 2007; Song et al., 2020a,b).

The discovery of this special structure not only brings new insights to the knowledge of the brain but also describes the "source" regulating the physicochemical property and biological composition of the CSF under different physiological or pathological conditions (Song et al., 2019). Moreover, it provides the morphological evidence of systemic effects after interventions via body fluids (Song et al., 2019). However, these studies were only in rats, the presence or absence of this nucleus has not been proven in primates. The presence of the CSF-contacting nucleus in primates has implications to provide the structural basis for brain-CSF communication and help to understand the neurohumoral regulatory mechanisms in humans.

The present study is the first to describe the CSF-contacting neurons distribution, their nearby structures, 3D spatial morphology, and the synaptic and non-synaptic transmissions between the CSF and the brain parenchyma in primates. This study will provide scientific evidence and theoretical basis of the CSF-contacting nucleus in the primate's brain.

\section{MATERIALS AND METHODS}

\section{Experimental Animals}

Adult male rhesus monkeys (average weight of $5.64 \pm 2.10 \mathrm{~kg}$ ) were acquired from the Kunming Primate Research Center of the Chinese Academy of Sciences, license number SCXK (Dian) K2017-0003. All the animal procedures were performed in accordance with the Guide for Care and Use of Laboratory Animals and were approved by the Institutional Animal Care and Use Committee (IACUC) of the Kunming Institute of Zoology, Chinese Academy of Sciences. The animals were singly housed $(0.80 \times 0.80 \times 0.80 \mathrm{~m})$ in a controlled environment (temperature: $18-26^{\circ} \mathrm{C}$; humidity: $40-70 \%$ ), with $12 \mathrm{~h}$ light $/ 12 \mathrm{~h}$ dark cycle.

\section{Tracer Administration}

Monkeys were anesthetized with ketamine $(10 \mathrm{mg} / \mathrm{kg}$, i.m.), and the head was fixed on a stereotaxic instrument. $50 \mu \mathrm{l} 30 \%$ CB-HRP (Sigma, United States), a specific tracer of the CSFcontacting nucleus through the ventricular system, was injected into the LV according to the stereotaxic coordinates provided by Paxinos et al. (2000). The successful injection was confirmed by extracting the CSF using a microsyringe. After $48 \mathrm{~h}$, the monkeys were perfused.

\section{Perfusion and Fixation}

The animals were anesthetized with ketamine $(10 \mathrm{mg} / \mathrm{kg}$, i.m.), the thoracic cavity was opened, and the pericardium was cut to expose the heart. A perfusion needle was inserted into the heart left ventricle. The animals were transcardially perfused with $2000 \mathrm{~mL}$ of $0.9 \%$ saline to wash out the blood. After, $4000 \mathrm{~mL}$ of $4 \%$ paraformaldehyde solution was used for fixation. After perfusion, the brain and the spinal cord were dissected out and placed in a $4 \%$ paraformaldehyde solution for post-fixation for $48 \mathrm{~h}$ at $4^{\circ} \mathrm{C}$.

\section{Tissue Sectioning and Immunofluorescence}

After fixation, the brain and the spinal cord were immersed in a 30\% sucrose solution until it sank to the bottom. Serial sections (50 $\mu \mathrm{m}$ thick) of the brain and the spinal cord were prepared using a freezing microtome (Leica, Germany). The sections were collected in phosphate buffer saline (PBS) for immunofluorescence staining. The sections were incubated with anti-CB primary antibody (1:600 dilution, List Biological Labs) at $4^{\circ} \mathrm{C}$ for overnight, followed by incubation with donkey anti-goat Alexa Fluor 594 secondary antibody (1:200 dilution, Life Technologies). Some of the sections were retained for CB-HRP/NeuN double immunofluorescence staining. The CB-HRP stained sections were incubated with anti-NeuN primary antibody (1:50 dilution, Cell Signaling Technology) at $4^{\circ} \mathrm{C}$ overnight, followed by incubation with donkey anti-rabbit Alexa Fluor 488 secondary antibody (1:200 dilution, Life Technologies). The sections were then mounted in sequence on the slides and counterstained with DAPI before covering with coverslips. The sections were observed and images were captured on a confocal microscope (Zeiss, Germany) using uniform parameters. After capturing the images, some sections were reused for Nissl staining to observe the neural morphology and Nissl body distributions. 


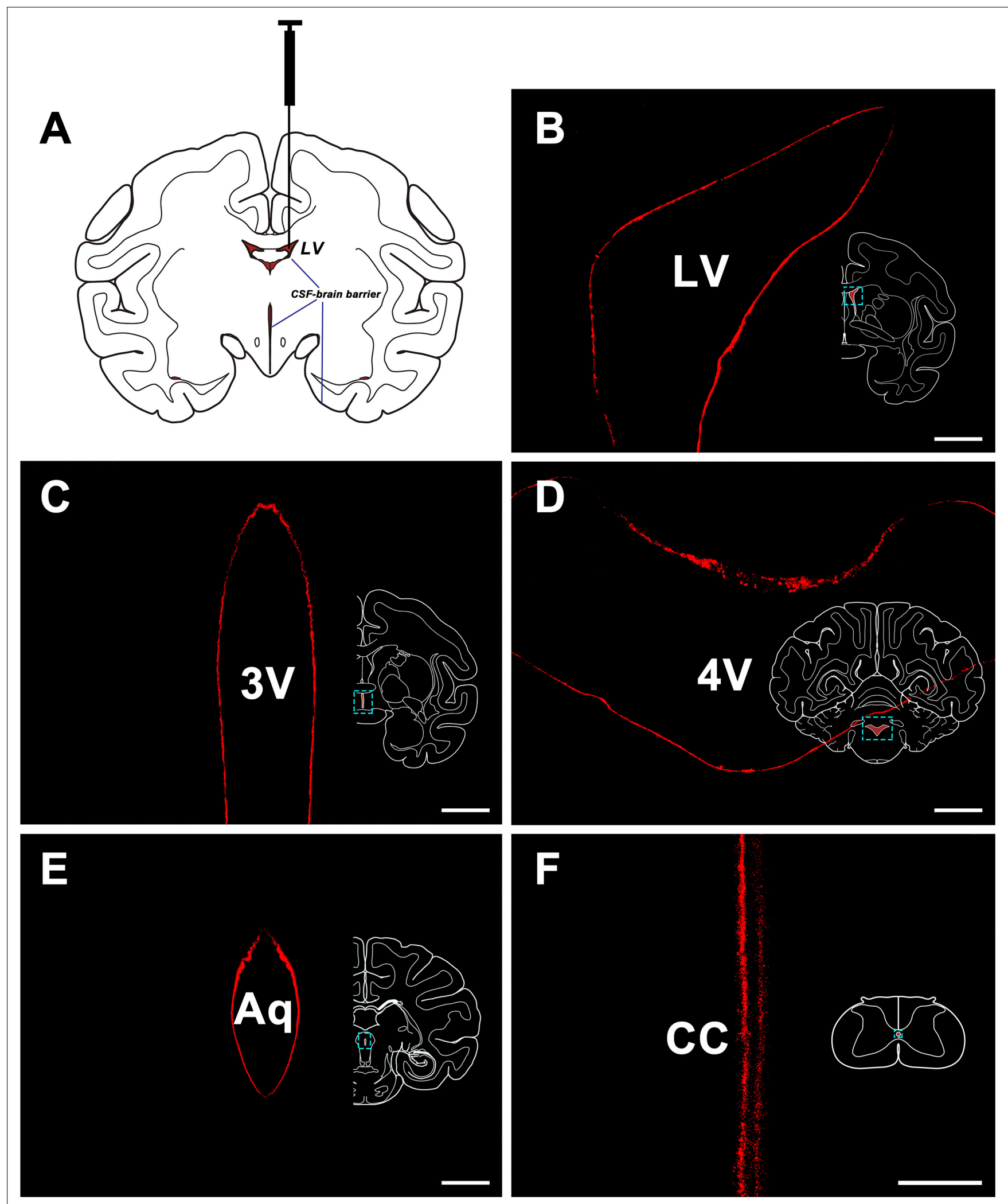

FIGURE 1 | CB-HRP flow in the ventricular system. (A) Schematic of the lateral ventricle injection. (B-F) CB-HRP immunofluorescence (red) on the ventricular wall. Bar $=0.5 \mathrm{~mm}$ (B-E) and $0.1 \mathrm{~mm}$ (F). LV, lateral ventricle; 3V, 3rd ventricle; Aq, aqueduct; 4V, 4th ventricle; CC, central canal. 


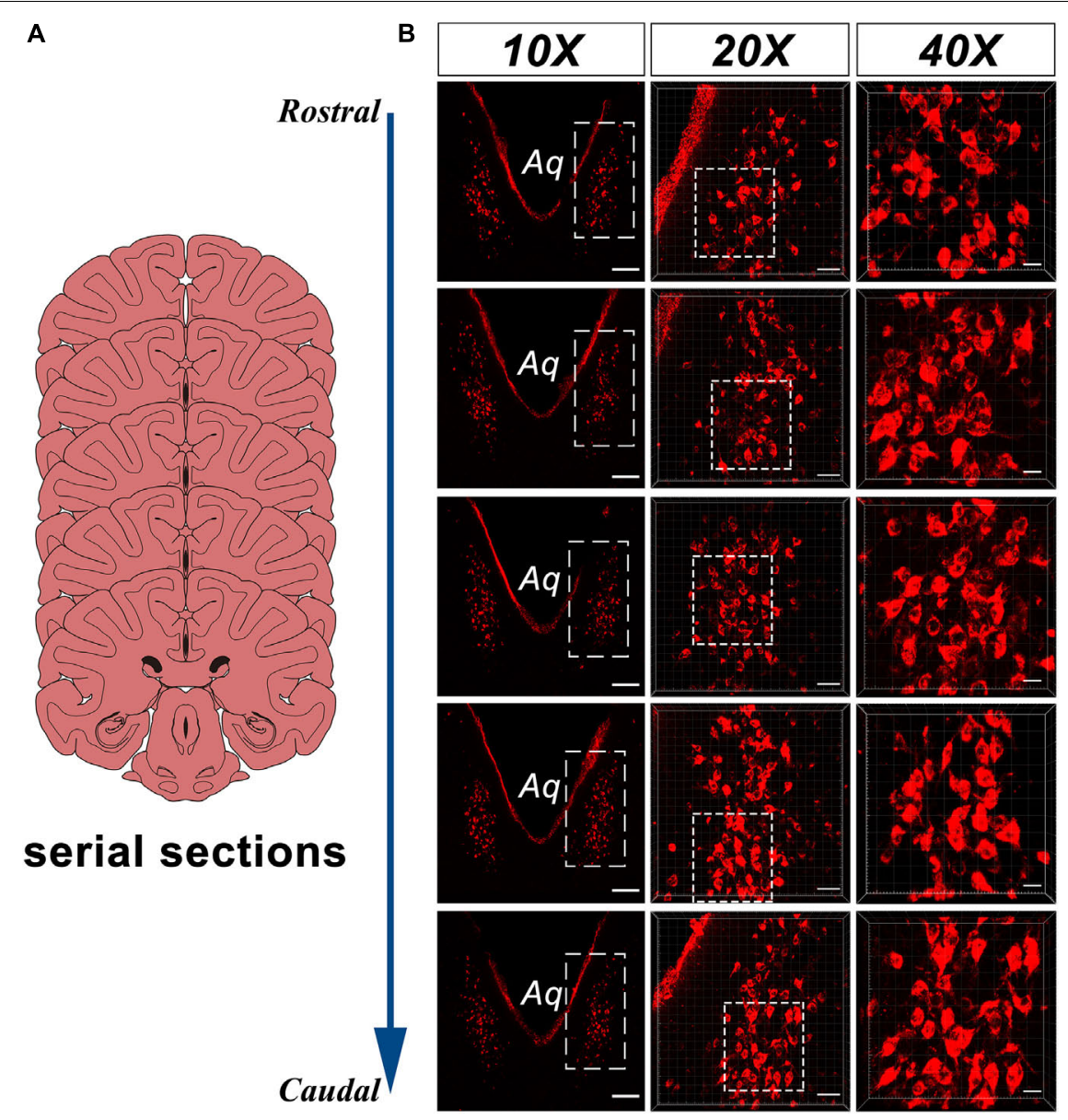

FIGURE 2 | CB-HRP positive neurons of the CSF-contacting nucleus in the parenchyma of the rhesus monkey brain. (A) Schematics of the serial sections, (B) CSF-contacting nucleus in the serial sections. $10 \times$ is the lower magnification. $20 \times$ and $40 \times$ are the higher magnification of the boxed regions. Aq, aqueduct. Bar $=200 \mu \mathrm{m}$ in $10 \times$, Bar $=50 \mu \mathrm{m}$ in $20 x$, Bar $=20 \mu \mathrm{m}$ in $40 x$.

\section{CSF-Contacting Nucleus Mapping and 3D Reconstruction}

The neurons in the CSF-contacting nucleus were mapped using the Adobe Illustrator software (Adobe, United States). The outline of the brain sections and the landmarks were illustrated and the neurons in the CSF-contacting nucleus were plotted into separate graphical layers. The brain serial sections and the CSF-contacting nucleus were $3 \mathrm{D}$ reconstructed using the Imaris software version 8.4.1 (Bitplane, United States). The "surface" module was used for rendering the brain surface and the CSFcontacting nucleus. The brain surface was colored blue while the CSF-contacting nucleus was colored yellow.

\section{Visualization of the Synaptic and Non-synaptic Structures of the CSF-Contacting Nucleus in the Monkey Brain}

The brain segment of the CSF-contacting nucleus was sliced using a vibratome (Leica, Germany) at $300 \mu \mathrm{m}$. CB-HRP staining was performed using the tetramethylbenzidine-sodium tungstate (TMB-ST) method (Gu et al., 1992; Lu et al., 2008). The CSF-contacting nucleus and the ventricle wall were isolated. Transmission and scanning electron microscopy were used to observe the synaptic and non-synaptic connections. (1) For transmission electron microscopy, the sections were immersed in a $2.5 \%$ glutaraldehyde solution overnight at $4^{\circ} \mathrm{C}$ and then postfixed in a $1 \% \mathrm{OsO}_{4}$ solution for $2 \mathrm{~h}$ at $4^{\circ} \mathrm{C}$. After, the sections were dehydrated using serial ethanol and embedded in Epon 812 resin. Ultra-thin sections (60 nm thick) were cut using an ultramicrotome (Leica EM UC7), stained with 2\% uranyl acetate and lead citrate, and observed under an electron microscope (JEM 1400 Plus, Japan). (2) For scanning electron microscopy, the sections were postfixed in $1 \%$ osmic acid for $2 \mathrm{~h}$. After, the sections were washed with distilled water three times ( 2 min each) and dehydrated using serial ethanol. The sections were then placed in isoamyl acetate and $\mathrm{CO}_{2}$ for critical point drying. The sections were placed on the stage using the conducting resin for spraying. The ultrastructure of the ventricle wall was observed using a scanning electron microscope (Teneo VS, United States). 

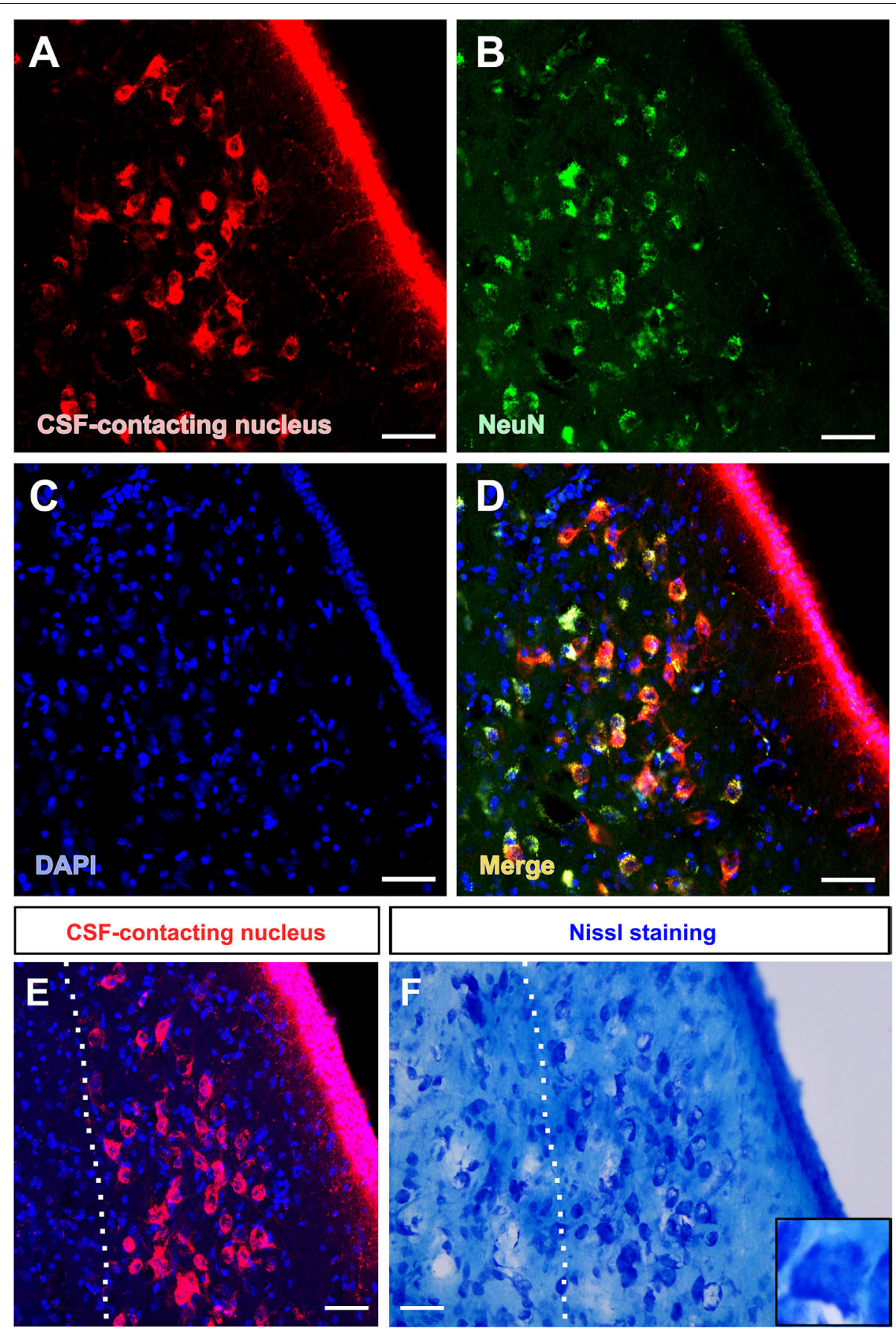

FIGURE 3 | Morphological features of the CSF-contacting nucleus in the rhesus monkey brain under light microscopy. (A) Neurons in the CSF-contacting nucleus (red), (B) Neuronal marker-NeuN (green), (C) DAPI (blue), (D) Co-labeling of the CSF-contacting neurons and NeuN (yellow). (E,F) The CSF-contacting nucleus (E) and its corresponding Nissl staining (F). Bar $=50 \mu \mathrm{m}$.

\section{RESULTS}

\section{CB-HRP Flow Within the Ventricular System and Brain Parenchyma Labeling}

The CB-HRP (red-fluorescence) was confined to the ventricular system and formed a clear outline of the lateral ventricle (LV), the third ventricle $(3 \mathrm{~V})$, the aqueduct $(\mathrm{Aq})$, the fourth ventricle $(4 \mathrm{~V})$ of the brain, and the central canal (CC) of the spinal cord. The nearby structures are not labeled suggesting that there is no leakage of the tracer (Figure 1).

In the caudal part of the ventral gray of the aqueduct and the rostral part of the $4 \mathrm{~V}$ floor of the monkey brain, a large number 


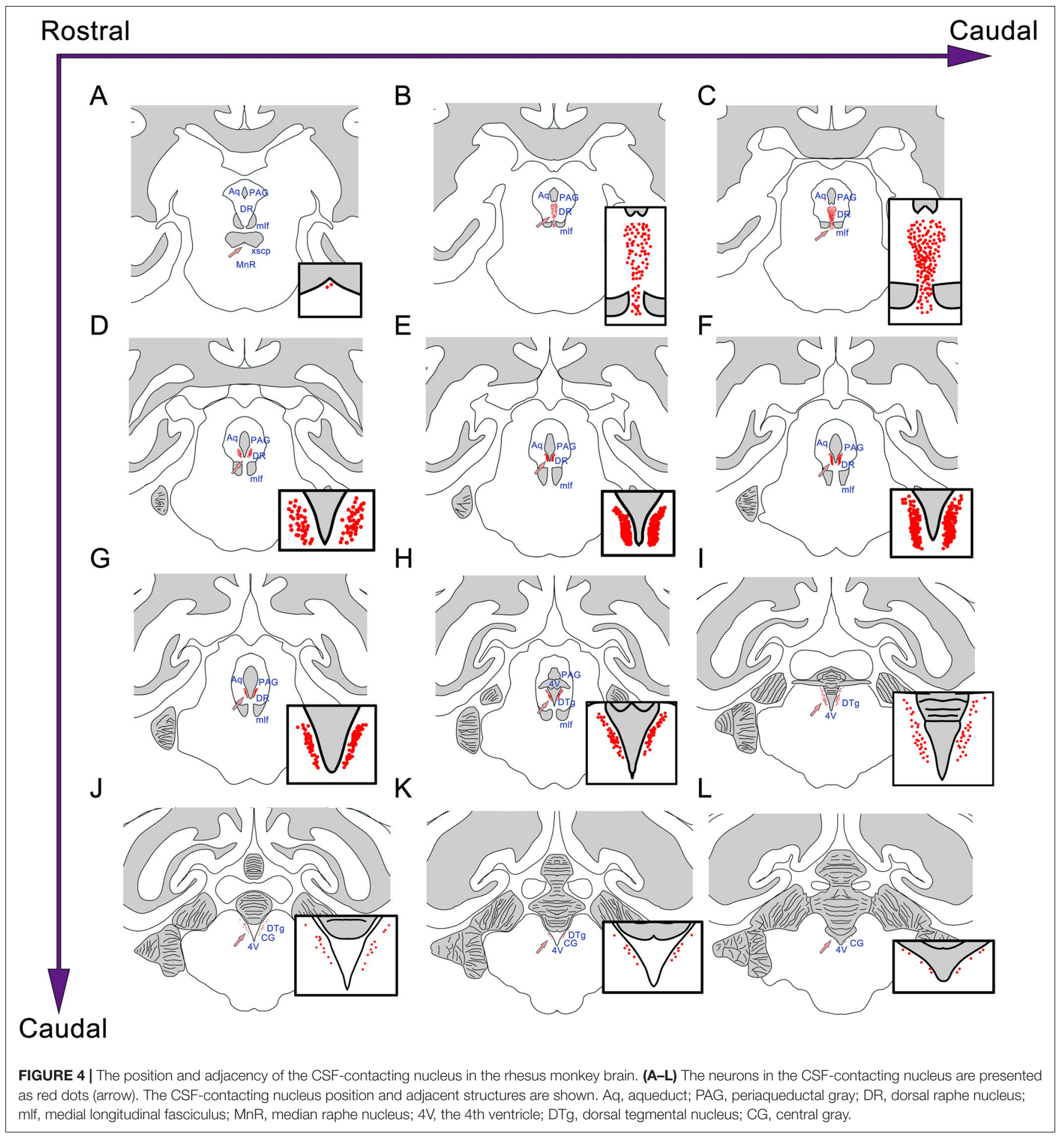

of CB-HRP positive neurons is located at consistent regions in the serial sections and form an independent cluster. The somata of these neurons appear to be fusiform or polygonal in shape and are easily distinguished from the nearby, unlabeled structures (Figure 2). These CB-HRP positive neurons are cerebrospinal fluid-contacting neurons.
The somata of the CSF-contacting neurons are co-labeled with NeuN, a neuron marker. Further, Nissl staining reveals that the neurons in the nucleus have abundant Nissl bodies. The staining is clear and the CSF-contacting neurons are brightly stained. The somata are slightly larger than the nearby neurons and the nucleolus is clear and evident (Figure 3). 

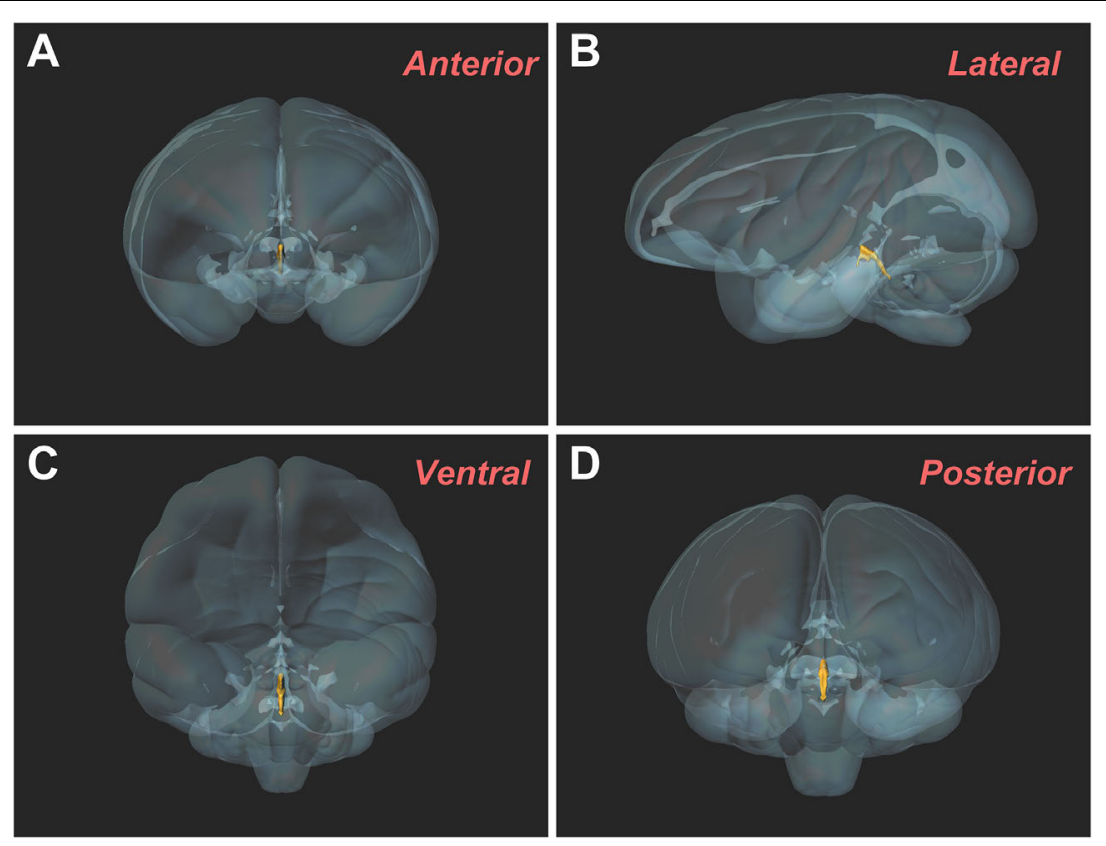

FIGURE 5 | Three-dimensional morphology of the CSF-contacting nucleus in the rhesus monkey brain from different perspectives. (A) Anterior view; (B) Lateral view; (C) Ventral view; (D) Posterior view.
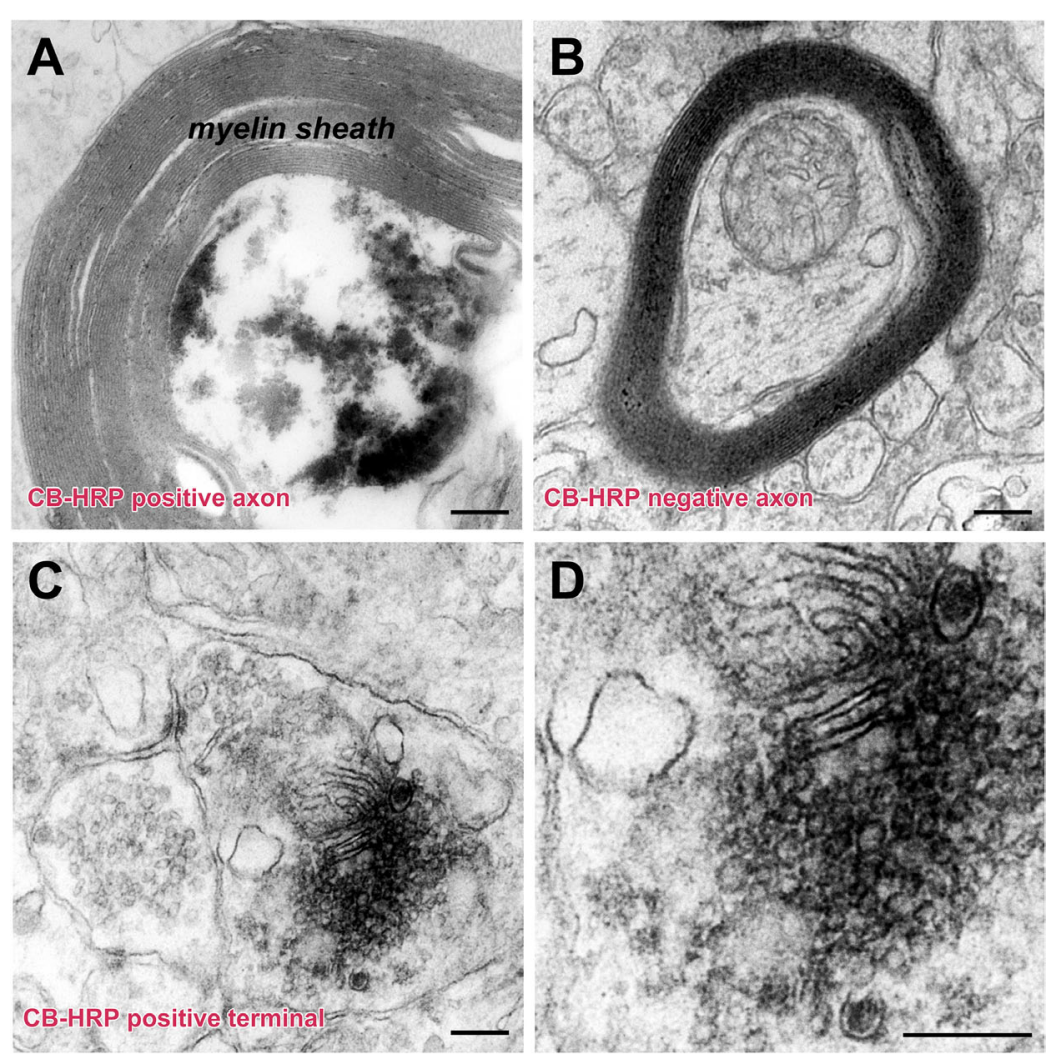

FIGURE 6 | The ultrastructure of the CSF-contacting neuron's axon and terminal in the rhesus monkey brain. (A,B) CB-HRP positive CSF-contacting neuron axon (A), the non-CSF-contacting neuron does not contain positive electron-dense deposits (B). (C,D) A CB-HRP positive CSF-contacting neuron terminal containing abundant synaptic vesicles (C); (D) is a higher magnification of (C). Bar $=200 \mathrm{~nm}$. 


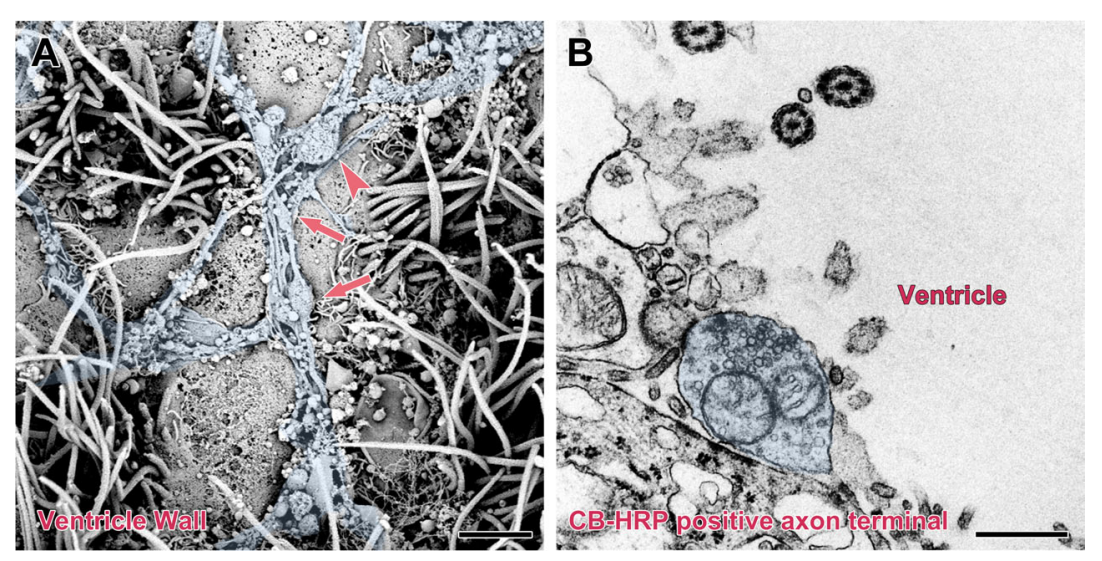

FIGURE 7 | Non-synaptic connections of the CSF-contacting neuron with CSF in the rhesus monkey brain. (A) Scanning electron microscopy reveals a large number of neural fibers (blue) with local varicosities $(\uparrow)$ and boutons $(\mathbf{\Lambda})$ located on the surface of the ventricle wall. (B) Transmission electron microscopy reveals a CB-HRP positive CSF-contacting neuron terminal (blue) stretching into the ventricle and contacting the CSF. Bar = $200 \mathrm{~nm}$ in (A) and $500 \mathrm{~nm}$ in (B).
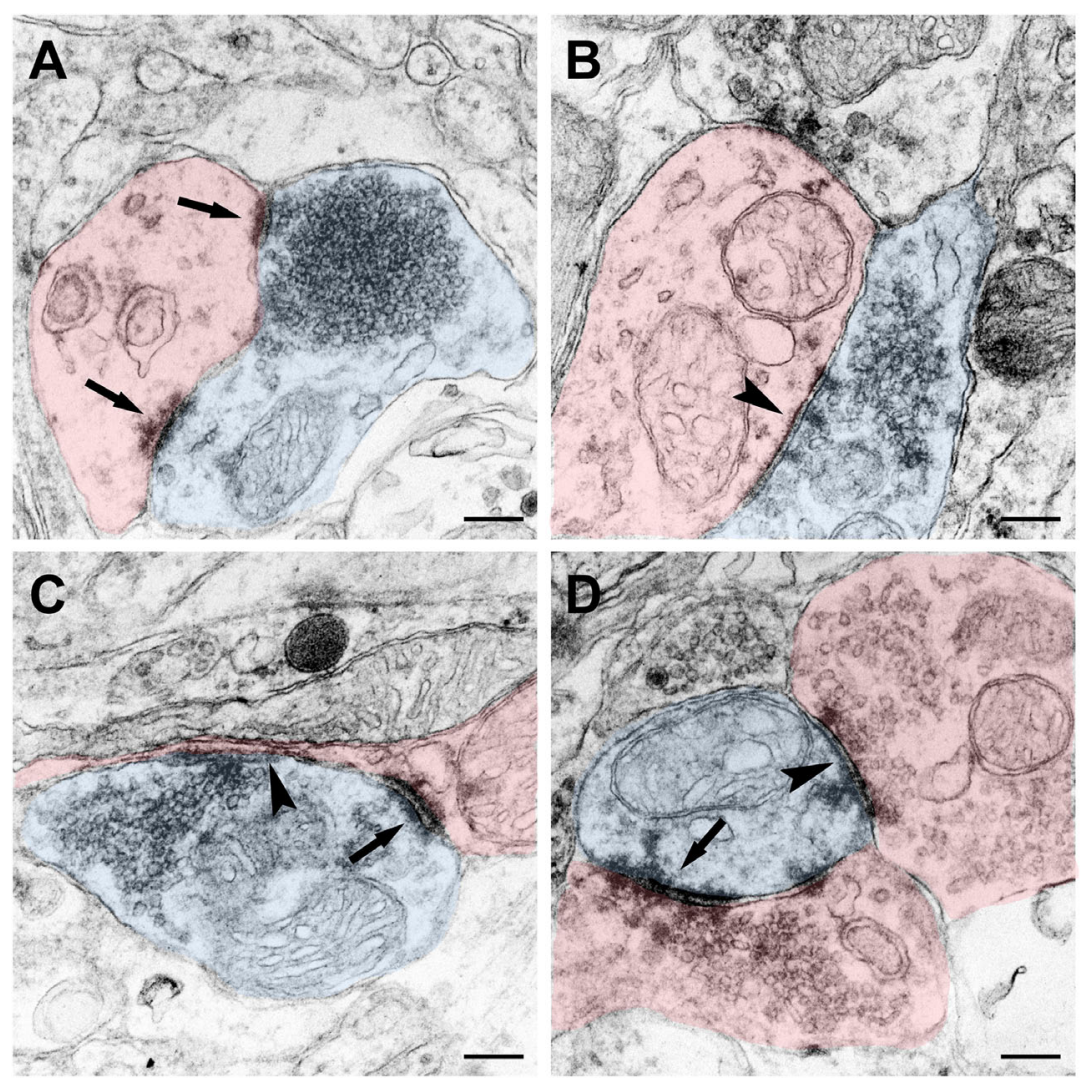

FIGURE 8 | Synaptic connections of the CSF-contacting neuron (blue) with the non-CSF-contacting neuron (red) in the rhesus monkey brain. (A) The asymmetrical synapse of the CSF-contacting neuron $\rightarrow$ non-CSF-contacting neuron ( $\uparrow$ ); (B) Symmetrical synapse of the CSF-contacting neuron $\rightarrow$ non-CSF-contacting neuron $(\mathbf{\Lambda})$;

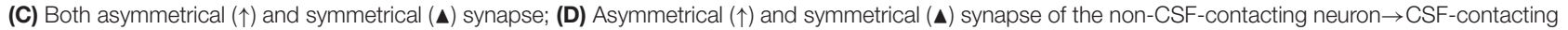
neuron. Bar $=200 \mathrm{~nm}$. 


\section{Position and Adjacency of the CSF-Contacting Nucleus in the Rhesus Monkey Brain}

Similar to the distribution of the CSF-contacting nucleus in rats, the nucleus in the monkey brain is located at the ventral gray of the lower part of the aqueduct and the upper part of the $4 \mathrm{~V}$ floor. The rostral part of the CSF-contacting nucleus is deep in the brain parenchyma and begins ventrally to the decussation of the superior cerebellar peduncle (xscp). The core of the CSFcontacting nucleus is symmetrical and ventral to the aqueduct. In this part, the nucleus is near to mlf, DR, and PAG. The caudal part of the nucleus is located in the ventral gray of the $4 \mathrm{~V}$ floor, near to the dorsal tegmental nucleus (DTg) and central gray (CG) (Figure 4).

\section{Three-Dimensional Reconstruction of the CSF-Contacting Nucleus}

The three-dimensional morphology of the CSF-contacting nucleus in the monkey brain was reconstructed using Imaris software. It shows a clear spatial morphology within the brain with definite spatial boundaries and specific location. The nucleus exists independently and consistently in the pons, including the isthmic region. The spatial morphology appears as a rivet-like shape (Figure 5).

\section{Ultrastructure of the CSF-Contacting Nucleus}

The injected CB-HRP it is transported along the axons of the CSF-contacting neurons. The CB-HRP electron-dense areas can be detected under electron microscopy and the CB-HRP positive axons are covered by a thick myelin sheath. The CB-HRP negative axons show no electron-dense areas. The CSF-contacting neuron terminal contains synaptic vesicles, which are round and flattened. Some of the vesicles have a dense core (Figure 6).

The surface of the brain ventricle was observed under the scanning electron microscope. Under the flagellum of the ependymal cells surface, numerous nerve fibers are visible. These nerve fibers have many inflated structures on the segments or terminals. Using transmission electron microscopy, we observed that the nerve fibers are the CB-HRP positive terminals of the CSF-contacting nucleus, which stretch into the ventricular system and contact the CSF forming the non-synaptic connections. The terminals contain many vesicles (Figure 7).

The terminal of the CSF-contacting neurons can be a presynaptic structure and form Gray I type excitatory (asymmetrical) or Gray II type inhibitory (symmetrical) synapses. Meanwhile, the terminals can also be post-synaptic structures and receive input from other neurons by Gray I or Gray II synaptic forms (Figure 8).

\section{DISCUSSION}

Our previous studies (Zhang et al., 2003; Song et al., 2019) in rodents confirmed that after CB-HRP injection into the CSF of the unilateral ventricular system, it can only flow to the contra-lateral $\mathrm{LV}, 3 \mathrm{~V}, \mathrm{Aq}, 4 \mathrm{~V}, \mathrm{CC}$ of the spinal cord and subarachnoid space. The tracer is localized on the walls of these structures and forms a clear outline. The present study in rhesus monkey confirms that the CB-HRP can only flow within the ventricular system and cannot pass through the CSF-brain barrier and leak into the brain parenchyma. Thus, the neurons whose processes stretch into the CSF can be labeled by CB-HRP. Therefore, the CB-HRP positive neurons observed in our study can only be the CSF-contacting neurons. The reason is that it has been proved repeatedly that there is an objective existence of the CSF-brain barrier.

Naming a nucleus in the brain is a very serious scientific question. It must satisfy strict naming conditions (Moore et al., 2006; Blumenfeld, 2010; Purves, 2012). (1) Similar morphology and functions: In the present study, all of the CB-HRP positive neurons are CSF-contacting neurons whose functions are specified for information connection between the brain and the CSF. (2) Similar location and occupy certain space: The CB-HRP positive neurons are located in the ventral gray of the aqueduct and $4 \mathrm{~V}$ floor, form an independent cluster and are significantly different from the nearby structures. The three-dimensional reconstruction results show that the entire morphology of the nucleus presents "rivet-like" shape and occupies a certain spatial volume. (3) The distribution characteristics have an evolutionary similarity: In both, rodents as well as in primates, the CSF-contacting nucleus initiates ventral of the xscp and ends at the ventral gray of the $4 \mathrm{~V}$ floor. The core of the nucleus is located at the ventral gray of the $\mathrm{Aq}$ and the nearby structures are DR, PAG, and mlf. The CSF-contacting nucleus in the monkey brain is larger than that in the rats, however, the position and shape are similar. The morphology of the nucleus is highly homologous between the two species. Therefore, we have sufficient evidence suggesting that the CSF-contacting nucleus exists objectively in primates.

The animal body is composed of not only parenchymal organs, but also includes fluids such as brain extracellular fluid, plasma, and CSF. It is believed that the body fluids are modified by the brain for specific functions. For example, neurons release to nearby extracellular fluid through autocrine or paracrine way to complete intercellular communication (Lozic et al., 2018). Hypothalamic pituitary portal system regulates systemic function via releasing neurohormone into blood (Clarke, 2015). However, the cerebrospinal fluid (CSF), an important part of the body, has not been reported to be regulated by a certain nucleus before.

Our study shows that the CSF-contacting nucleus is also existed in primates. Its position is in the pons, including the isthmic region. Although located in the brain parenchyma, it is different from other nuclei in the brain. Its characteristic is that the cell body is in the brain parenchyma, and the processes extend in the CSF, which indicates the characteristics of peripheral nerves similar to 12 pairs of cranial nerves. We recommend listing it as the XIII pair of cranial nucleus, whose basic function is to mediate the information transmission between brain and CSF. This is also very important and necessary in the body, which is of significance to the completing of innervation in the organism. Certainly, deep research is needed to address the further conclusions. 


\section{DATA AVAILABILITY STATEMENT}

All datasets presented in this study are included in the article/supplementary material.

\section{ETHICS STATEMENT}

The animal study was reviewed and approved by the Institutional Animal Care and Use Committee (IACUC) of the Kunming Institute of Zoology, Chinese Academy of Sciences.

\section{AUTHOR CONTRIBUTIONS}

S-YS and L-CZ designed the study and prepared the manuscript. S-YS, X-MZ, C-JS, L-LL, JH, and J-LC conducted the studies. All authors read and approved the manuscript.

\section{REFERENCES}

Agnati, L. F., Marcoli, M., Leo, G., Maura, G., and Guidolin, D. (2017). Homeostasis and the concept of 'interstitial fluids hierarchy': relevance of cerebrospinal fluid sodium concentrations and brain temperature control (Review). Intern. J. Mol. Med. 39, 487-497. doi: 10.3892/ijmm.2017. 2874

Blumenfeld, H. (2010). Neuroanatomy Through Clinical Cases, 2nd Edn, Sunderland, MA: Sinauer Associates.

Clarke, I. J. (2015). Hypothalamus as an endocrine organ. Comprehens. Physiol. 5, 217-253. doi: 10.1002/cphy.c140019

Gu, Y., Chen, Y., and Ye, L. (1992). Electron microscopical demonstration of horseradish peroxidase by use of tetramethylbenzidine as chromogen and sodium tungstate as stabilizer (TMB-ST method): a tracing method with high sensitivity and well preserved ultrastructural tissue. J. Neurosci. Methods 42, 1-10. doi: 10.1016/0165-0270(92)90129-2

Liang, D., Zhang, L. C., Qin, C. W., and Zeng, Y. M. (2007). The ultrastructure of the distal CSF-contacting neurons in the dorsal raphe and the relationships with their surrounding tissues. Acta Anat. Sinica 38, 410-413.

Lozic, M., Sarenac, O., Murphy, D., and Japundzic-Zigon, N. (2018). Vasopressin, central autonomic control and blood pressure regulation. Curr. Hypert. Rep. 20:11.

Lu, X., Geng, X., Zhang, L., and Zeng, Y. (2008). The methodology for labeling the distal cerebrospinal fluid-contacting neurons in rats. J. Neurosci. Methods 168, 98-103. doi: 10.1016/j.jneumeth.2007.09.033

Moore, K. L., Dalley, A. F., and Agur, A. M. R. (2006). Clinically Oriented Anatomy, 5th Edn, Philadelphia: Lippincott Williams \& Wilkins.

Paxinos, G., Huang, X. F., and Toga, A. W. (2000). The Rhesus Monkey Brain In Stereotaxic Coordinates. San Diego, CA: Academic Press.

Purves, D. (2012). Neuroscience, 5th Edn, Sunderland, MA: Sinauer Associates.

Rambeck, B., Jurgens, U. H., May, T. W., Pannek, H. W., Behne, F., Ebner, A., et al. (2006). Comparison of brain extracellular fluid, brain tissue, cerebrospinal fluid, and serum concentrations of antiepileptic drugs measured intraoperatively in patients with intractable epilepsy. Epilepsia 47, 681-694. doi: 10.1111/j.15281167.2006.00504.x

\section{FUNDING}

This research was supported by the National Natural Science Foundation of China (Grant Nos. 81371243 and 81901131), the Natural Science Foundation of Jiangsu Province (Grant No. BK20190987), and Chinese Postdoctoral Science Foundation (Grant No. 2018M642328).

\section{ACKNOWLEDGMENTS}

We thank all members of L-CZ group at Xuzhou Medical University. We also thank Ying-Qi Guo in Kunming Institute of Zoology of Chinese Academy of Sciences for her help in electron microscopy experiments. This manuscript has been released as a pre-print at Research Square (Song et al., 2020c).

Song, S. Y., Li, Y., Zhai, X. M., Li, Y. H., Bao, C. Y., Shan, C. J., et al. (2020a). Connection Input mapping and $3 \mathrm{D}$ reconstruction of the brainstem and spinal cord projections to the CSF-contacting nucleus. Front. Neural Circ. 14:11. doi: 10.3389/fnana.2019.00011

Song, S. Y., Li, Y., Zhai, X. M., Li, Y. H., Bao, C. Y., Shan, C. J., et al. (2020b). Monosynaptic input mapping of diencephalic projections to the cerebrospinal fluid-contacting nucleus in the rat. Front. Neuroanat. 14:7. doi: 10.3389/fnana. 2019.00007

Song, S. Y., Li, Y., Zhai, X. M., Lu, L. L., Li, Y. H., Bao, C. Y., et al. (2020c). A special cranial nucleus (CSF-contacting nucleus) in primates. Research Square [Preprint]. doi: 10.21203/rs.2.21682/v1

Song, S. Y., Li, Y. H., Bao, C. Y., Li, Y., Yin, P. C., Hong, J., et al. (2019). Stereotaxic coordinates and morphological characterization of a unique nucleus (CSFContacting Nucleus) in rat. Front. Neuroanat. 13:47. doi: 10.3389/fnana.2019. 00047

Song, S. Y., and Zhang, L. C. (2018). The establishment of a CSF-contacting nucleus "Knockout" model animal. Front. Neuroanat. 12:22. doi: 10.3389/fnana.2019. 00022

Zhang, L. C., Wang, M., and Zhang, F. (1994). A tracing study on neuron contacting cerebrospinal fluid in rat. Chin. J. Anat. 1, 37-40.

Zhang, L. C., Zeng, Y. M., Ting, J., Cao, J. P., and Wang, M. S. (2003). The distributions and signaling directions of the cerebrospinal fluid contacting neurons in the parenchyma of a rat brain. Brain Res. 989, 1-8. doi: 10.1016/ s0006-8993(03)03123-8

Conflict of Interest: The authors declare that the research was conducted in the absence of any commercial or financial relationships that could be construed as a potential conflict of interest.

Copyright (c) 2020 Song, Zhai, Shan, Lu, Hong, Cao and Zhang. This is an open-access article distributed under the terms of the Creative Commons Attribution License (CC BY). The use, distribution or reproduction in other forums is permitted, provided the original author(s) and the copyright owner(s) are credited and that the original publication in this journal is cited, in accordance with accepted academic practice. No use, distribution or reproduction is permitted which does not comply with these terms. 